\title{
Diagnostic et dépistage anténatals au fil de l'actualité et de sa transversalité
}

\section{Antenatal diagnosis and screening throughout updating and transversality}

\author{
C. Donner \\ (C) Lavoisier SAS 2020
}

La généralisation dans le monde occidental des consultations prénatales dans les années 1950 et les nombreux progrès techniques en médecine ont ouvert la possibilité de s'intéresser aux pathologies à « haut risque » fœtal. Même si quelques cas de prélèvements de liquide amniotique avaient été rapportés au XIX ${ }^{\mathrm{e}}$ siècle et uniquement dans des tentatives thérapeutiques quasi désespérées, il faudra attendre les années 1960 pour que cette procédure soit proposée dans un but diagnostique. L'amniocentèse est alors proposée dans la surveillance de l'alloimmunisation rhésus, pour la détermination du sexe fœtal par étude cytologique des cellules présentes dans le liquide amniotique et ensuite par la mise en évidence de la chromatine de Barr, ce qui a changé l'abord des maladies héréditaires liées au chromosome $\mathrm{X}$ telles l'hémophilie ou la dystrophie musculaire. Ensuite plusieurs auteurs ont démontré la possibilité de détection anténatale de certaines maladies métaboliques. L'étape déterminante fut la possibilité de cultiver et d'étudier le caryotype des cellules fœtales présentes dans le liquide amniotique et donc de diagnostiquer les anomalies du caryotype tel le syndrome de Down à la fin des années 1960. À la même époque, la biopsie de villosités est envisagée ; cependant, les nombreux échecs de la technique et un risque de perte de la grossesse supérieur à celui de l'amniocentèse postposeront son développement d'une dizaine d'années. Les ultrasons étaient de qualité médiocre, et c'est en 1977 que le premier diagnostic anténatal d'anencéphalie a été réalisé par échographie. Depuis, des progrès techniques spectaculaires en imagerie ont permis la visualisation du fœtus in utero, de mieux comprendre son évolution et aussi le diagnostic de nombreuses pathologies congénitales et d'anomalies du développement fœtal.

\footnotetext{
C. Donner $(\bowtie)$
}

Clinique d'échographie et médecine fœtale,

Hôpital Erasme, Université Libre de Bruxelles,

Route de Lennik, 808, B-1070 Bruxelles, Belgique

e-mail : cdonner@ulb.ac.be
L'introduction de l'échographie et la possibilité d'un prélèvement échoguidé de liquide amniotique, de villosités choriales et de sang fœtal au début des années 1980 ont considérablement amélioré l'efficacité et l'innocuité des différents modes de prélèvement. Ces techniques ont également permis in utero et pour quelques pathologies ciblées des prises en charge thérapeutiques ; de la transfusion in utero, aux drainages de liquide amniotique ou de collection liquidienne, à la mise en place de shunts et à l'utilisation du fœtoscope et du laser.

Parallèlement à ces améliorations de l'imagerie anténatale, la recherche d'infections congénitales, les progrès de la génétique et de la biologie moléculaire, les nouvelles techniques génomiques ont élargi la liste des indications du diagnostic anténatal et étendu les possibilités de dépistage non invasif. Cependant, ces dépistages et ces diagnostics de plus en plus précis complexifient les conseils donnés aux futurs parents et soulèvent de nouvelles questions. Dans le chemin à parcourir avec eux, l'évaluation d'un pronostic et les zones d'incertitudes restent parmi les étapes les plus complexes et souvent les plus douloureuses.

L'importance de l'impact émotionnel de l'annonce d'une augmentation de risque ou celle d'une mauvaise nouvelle a été mesurée au fil des années de pratique du dépistage et du diagnostic anténatal. Les parents viennent à l'échographie pour une première « rencontre » avec leur futur enfant et pour se représenter la vie fotale en temps réel, en 2, 3 ou 4 dimensions. L'échographie a cette particularité de remplir plusieurs rôles à la fois dans la même instantanéité : un moment de découverte heureuse pour les futurs parents et l'échographiste, mais aussi une possible indication de dépistage ou d'annonce d'un diagnostic d'une pathologie fœtale.

Une autre particularité du diagnostic anténatal est que la part dédiée au traitement reste faible et que le diagnostic anténatal est caractérisé par le large écart persistant entre la haute technicité des moyens utilisés et la dureté des solutions proposées qu'il s'agisse tant des interruptions médicales de grossesse que de la mise en place de soins palliatifs. 
L'accès progressif de l'ensemble de la population des femmes enceintes aux techniques de dépistage et de diagnostic anténataux nécessite une mobilisation et une actualisation permanente des connaissances pour tous les acteurs du champ périnatal.

Une approche multidisciplinaire s'est rapidement imposée mettant ensemble les connaissances des différents spécialistes impliqués dans ces situations complexes.

Les sages-femmes, à travers leurs savoirs complémentaires, ont enrichi l'accompagnement de ces femmes et de ces couples en situation difficile. L'écoute, la disponibilité, la bienveillance, l'anticipation, la transmission et le temps qui y sont consacrés sont la pierre angulaire du tandem formé par les sages-femmes et les obstétricien.ne.s.

Au-delà des progrès techniques et médicaux, l'approche inter- et transdisciplinaire s'est construite. L'annonce de nouvelles compliquées a nécessité l'apprentissage d'un accompagnement cohérent autour des couples. L'individualité de chaque situation, la grande variabilité contextuelle pour chaque femme enceinte et chaque couple, a mobilisé toutes les ressources aussi bien d'un point de vue médical qu'émotionnel. Cet accompagnement a ouvert la porte aux collaborations avec les acteurs du champ « psy » dans une idée d'amélioration constante de cette prise en charge parti- culière. Ces collaborations se sont affinées grâce au travail commun et au fait de découvrir ensemble la spécificité de cette médecine anténatale jusqu'alors peu connue. L'apport des témoignages des parents a été et reste fondamental pour continuer à progresser dans cet apprentissage.

Le domaine du dépistage et du diagnostic anténatals est en constante évolution. L'interprétation des résultats et la réflexion autour de leur transmission aux parents font l'objet d'une remise à jour permanente et d'une réflexion éthique.

Nous avons voulu presque dix ans après deux numéros de la Revue de médecine périnatale consacrés au diagnostic anténatal et plus récemment aux avancées du dépistage réaborder ce thème. Dans cette nouvelle mouture de la revue nommée " Périnatalité », nous avons tenté d'en illustrer différentes facettes à la fois dans une actualisation des connaissances mais aussi dans une approche transdisciplinaire. Les aspects proposés sont loin d'être exhaustifs, et d'autres pourraient faire l'objet d'un nouveau numéro.

Ce numéro de la revue a été pensé et écrit en grande partie pendant la crise sanitaire liée à la Covid-19. Malgré cela, nous espérons pouvoir transmettre l'enthousiasme et la curiosité toujours en éveil qui animent et portent les équipes de l'anténatal. Nous remercions vivement les auteur.e.s qui y ont participé. 